\title{
Altered cortical phase- and amplitude-coupling in Multiple Sclerosis
}

\author{
Marcus Siems ${ }^{1,2,3}$, Johannes Tünnerhoff ${ }^{1,4}$, Ulf Ziemann ${ }^{1,4}$ and Markus Siegel ${ }^{1,2,3}$
}

\section{Author affilitations:}

\footnotetext{
${ }^{1}$ Hertie Institute for Clinical Brain Research, University of Tübingen, Germany

${ }^{2}$ Centre for Integrative Neuroscience, University of Tübingen, Germany

${ }^{3}$ MEG Center, University of Tübingen, Germany

${ }^{4}$ Department of Neurology \& Stroke, University of Tübingen, Germany
}

Correspondence: Marcus Siems (m.siems@uke.de) \& Markus Siegel (markus.siegel@unituebingen.de)

Keywords: MEG, Multiple Sclerosis, functional connectivity, oscillation, amplitude-coupling, phase-coupling, classification, human connectome project

\begin{abstract}
Abbreviations: EDSS $=$ Expanded Disability Status Scale; MEG $=$ magnetoencephalography; MS = Multiple Sclerosis; MSFC = Multiple Sclerosis Functional Composite; RRMS = Relapsing-remitting Multiple Sclerosis
\end{abstract}

\section{Abstract}

Multiple Sclerosis is a demyelinating disease of the central nervous system that can result in cognitive decline and physical disability. However, related functional changes in large-scale brain interactions remain poorly understood and corresponding non-invasive biomarkers are sparse. Here, we measured magnetoencephalography in 17 relapsing-remitting Multiple Sclerosis patients at an early disease stage (median EDSS $=1.5$, range 0 to 3.5 ) and 17 healthy controls to investigate brain-wide phase- and amplitude-coupling of frequency specific neuronal activity. We developed a new analysis approach that combines dimensionality reduction, bootstrap aggregating and multivariate classification to identify changes of brain-wide coupling in Multiple Sclerosis. We identified systematic and nonredundant changes of both phase- and amplitude-coupling. Changes included both, increased and decreased neuronal coupling in wide-spread, bilateral neuronal networks across a broad range of frequencies. These changes allowed to successfully classify patients and controls with an accuracy of $84 \%$. Furthermore, classification confidence predicted behavioral scores of disease severity. Our results unravel systematic changes of large-scale neuronal coupling in Multiple Sclerosis and suggest non-invasive electrophysiological coupling measures as powerful biomarkers of Multiple Sclerosis. 


\section{Introduction}

Multiple Sclerosis (MS) is an inflammatory, demyelinating secondary neurodegenerative disease ${ }^{1}$. From early disease stages on and during disease progression ${ }^{2}$, accumulating white-matter lesions and cortical atrophy can lead to cognitive decline and physical disability $^{3}$. While gross white-matter damage itself is only weakly related to disease severity and duration ${ }^{4-6}$, fine-grained analyses of structural connectivity and neuronal coupling revealed that the location of affected white-matter tracts is pivotal ${ }^{6,7}$. Using Magnetic Resonance Imaging (MRI), Magnetoencephalography (MEG) and Electroencephalography (EEG), anatomical and functional network changes have been related to tissue pathology ${ }^{8-}$ ${ }^{10}$, cognitive deficits ${ }^{7,11-21}$, clinical subtypes ${ }^{22}$ and disease duration ${ }^{23}$.

The high temporal resolution of MEG and EEG allows to characterize the frequency-specific coupling of neuronal activity, which may reflect ${ }^{24,25}$ and even mediate ${ }^{26}$ interactions in largescale brain networks. Accordingly, electrophysiological studies in MS patients have revealed changes of neuronal coupling in specific frequency ranges ${ }^{10,13,16,17,21}$. While these studies have largely focused on phase-coupling as a measure of functional connectivity, recent findings have highlighted amplitude-coupling as another mode of neuronal interactions that may provide robust connectivity information non-redundant to phase-coupling ${ }^{21,27-32}$. Thus, the central goal of this study was first, to jointly identify features of cortical phase- and amplitude-coupling that can dissociate MS patients from healthy controls and second, to investigate if these features differ between amplitude- and phase-coupling.

The direct comparison of brain-wide coupling between populations is generally impeded by the large-number of connections and corresponding comparisons ${ }^{33}$. Thus, another central goal of this study was to develop a novel analysis approach that combines dimensionality reduction, bootstrap aggregating and classification to compare brain-wide coupling data between populations.

We applied this approach to resting-state MEG recordings of relapsing-remitting MS (RRMS) patients at an early disease stage (median EDSS $=1.5$, range 0 to 3.5$)^{34,35}$ and of healthy controls. We found that cortical phase- and amplitude-coupling allowed to successfully classify (84\% correct) RRMS patients and controls. Importantly, both coupling measures showed significant and non-redundant changes of neuronal coupling over a broad range of frequencies and cortical networks. Our results highlight non-invasive electrophysiological coupling measures as powerful new biomarkers of Multiple Sclerosis. 


\section{Materials and methods}

\section{Subjects and dataset}

We analyzed MEG data from two datasets. The first dataset was recorded at the MEGCenter Tübingen and included eyes-open resting-state MEG measurements from 34 subjects. 17 of these subjects ( 8 female, mean age ( \pm std) $31.1 \pm 9.6$ years) were diagnosed with RRMS and 17 subjects were healthy controls ( 9 female, mean age $( \pm$ std) $28.4 \pm 4.2$ years, $p=0.30$ ). The patient group was measured prior to the first application of Tecfidera (dimethyl fumarate; BioGen Inc., Cambridge, MA, USA) with a median disease duration of 1 month (0 - 3 years interquartile range, maximum 11 years). Patients had no or mild to moderate neurological impairment, which was assessed with the Expanded Disability Status Scale $\left(n=16 \text {; median } E{ }^{2} S_{\text {total }}=1.5 \text {, range } 0 \text { to } 3.5\right)^{36}$ and the Multiple Sclerosis Functional Composite $\left(\mathrm{n}=13 \text {; median } \mathrm{MSFC}_{\text {totalz }}=-1.8 \text {, range }-0.3 \text { to }-3.3\right)^{37}$. All participants gave written informed consent in accord with the Declaration of Helsinki, and the study was approved by the ethics committee of the medical faculty of the University of Tübingen.

We collected 10 minutes of eyes-open resting-state MEG data per subject. The MEG was continuously recorded with a 275-channel whole-head system (Omega 2000, CTF Systems Inc., Port Coquitlam, Canada) in a magnetically shielded room. The head position was tracked using three head localization coils fixated at the nasion and the left and right preauricular points. MEG signals were recorded with $2343.75 \mathrm{~Hz}$ sampling frequency and down sampled to $1000 \mathrm{~Hz}$ offline.

A T1-weighted sagittal MRI was obtained from each participant to construct individual highresolution head models (MPRAGE sequence, TE $=2.18 \mathrm{~ms}, \mathrm{TR}=2300 \mathrm{~ms}, \mathrm{TI}=1100 \mathrm{~ms}$, flip angle $=9^{\circ}, 192$ slices, voxel size $=1 \times 1 \times 1 \mathrm{~mm}$ ). The subjects were scanned in a Siemens MAGNETOM Trio 3T scanner (Erlangen, Germany) with a 32-channel head coil.

The second dataset included 95 subjects from the publicly available human connectome project (HCP) $S 900$ release $^{38}$. Participants were healthy adults in the age range between 2235 years $\left(n_{22-25}=18, n_{26-30}=40, n_{31-35}=37\right)$. The HCP-sample included 45 females. The HCP-MEG data included three six-minute blocks of eyes-open resting-state MEG with short breaks in between measurements. Data were recorded with a whole-head Magnes 3600 scanner (4D Neuroimaging, San Diego, CA, USA) situated in a magnetically shielded room $^{38}$. Additionally, the HCP-subjects were scanned on a Siemens $3 T$ Skyra to acquire structural T1-weighted magnetic resonance images (MRI) with $0.7 \mathrm{~mm}$ isotropic resolution ${ }^{39}$. 


\section{Data preprocessing}

For the Tübingen-dataset, we first notch-filtered line noise at $50 \mathrm{~Hz}$ and at the first six harmonics (stop-band width: $1 \mathrm{~Hz}$ ). Second, we visually inspected the data for muscle-, eyeblink-, and technical artifacts (SQUID-jumps). We rejected corresponding time intervals and malfunctioning or noisy channels (mean: 1 channel; range: 0 to 3 channels). Third, we high-pass filtered the data at $0.5 \mathrm{~Hz}$ with a $4^{\text {th }}$-order zero-phase Butterworth filter and split the data into two frequency bands: a low frequency band from $0.5-30 \mathrm{~Hz}$ and a high frequency band with frequencies above $30 \mathrm{~Hz}$. For both frequency ranges, we separately performed independent component analysis (ICA $)^{40}$. This approach takes advantage of the distinct spectral profile of different physiological artifacts, such as muscle and eye-movement artifacts $^{41}$. For both frequency ranges, independent components were visually inspected and artifactual components were rejected according to their topology, time course and spectrum ${ }^{41,42}$. Finally, both frequency bands were recombined.

For the HCP-dataset we used the preprocessed data as provided by the HCP pipeline ${ }^{38}$. This included removal of noisy and malfunctioning channels, bad data segments and physiological artifacts by the iterative application of temporal and spatial independent component analysis ${ }^{38,43}$.

\section{Physical forward model and source modeling}

MEG sensors were aligned to the individual anatomy using FieldTrip ${ }^{44}$. We segmented the individual T1-weighted images and generated a single shell head model to compute the physical forward model ${ }^{45}$ for 457 equally spaced $(\sim 1.2 \mathrm{~cm}$ distance) source points spanning the cortex at $0.7 \mathrm{~cm}$ depth below the pial surface ${ }^{25,32,33}$. The source shell was generated in $\mathrm{MNI}$-space and non-linearly transformed to individual headspace. We co-registered the source coordinates, head model and MEG channels on the basis of the three head localization coils.

The sensor-level MEG data was projected to source space using linear beamforming ${ }^{46,47}$. This spatial filtering approach reconstructs activity of the sources of interest with unit gain while maximally suppressing contributions from other sources.

\section{Spectral analysis}

We generated time-frequency estimates of the time-domain MEG signal using Morlet wavelets $^{48}$. The bandwidth of the wavelets (1 spectral standard deviation) was set to 0.5 octaves with a temporal step-size of half the temporal standard deviation. We derived spectral estimates for 23 frequencies from 2.8 to $128 \mathrm{~Hz}$ in quarter-octave steps. 


\section{Neuronal coupling}

We estimated neuronal amplitude- and phase-coupling by means of amplitude envelope correlations of orthogonalized signals ${ }^{28}$ and the weighted phase lag index ${ }^{49}$, respectively. Importantly, both measures are insensitive to volume conduction and might relate to distinct functional mechanisms of cortical network interactions ${ }^{24,31,32,50}$. For the application of amplitude-coupling we used pairwise orthogonalization of the two complex signals at each time-point ${ }^{27,28}$ before correlating the log-transformed power envelopes.

For both metrics all subjects and frequency bands, we generated full and symmetric correlation matrices. For further analyses, we vectorized all unique connections of these correlation matrices. We refer to the resulting vectors as coupling profiles.

\section{Direct comparison of coupling}

For every connection $\left(n_{c}=104,196\right)$ in each frequency band $\left(n_{f}=23\right)$, we tested for group differences in coupling strength (two-tailed Mann-Whitney U-tests) and applied falsediscovery rate correction ${ }^{51}$ for multiple-comparison correction within each frequency. Further, we tested which group was more likely to show stronger coupling in each frequency band by testing the distribution of the sign of significant t-scores $(p<0.05$, corrected) against 0.5 using a binomial test. In other words, we tested the Null-hypothesis for the same probability of positive and negative signs. For the binominal statistic, we conservatively estimated the degrees of freedom at $\mathrm{df}=40$ as the rank of the forward model, i.e., the maximum amount of independently separable sources ${ }^{25,52}$. Furthermore, we FDR-corrected the results of the binomial tests across frequencies.

\section{Dimensionality reduction of coupling space}

In a first step, in the HCP dataset, for each frequency and coupling measure, we applied PCA to the coupling profiles across HCP subjects $\left(n_{\text {hcp }}=95\right)$ in order to identify components, i.e., networks of coupling that explain most variability across subjects. Next, we projected the coupling profiles of the Tübingen patient and control subject dataset into the PCA space. For each frequency and both coupling modes, we multiplied the z-scored coupling profiles of every subject with the component eigenvectors of the PCA. In each frequency band, we used the 30 principal components with the highest eigenvalues, which resulted in 690 feature dimensions per coupling measure (coupling components). 


\section{Feature bagging and group classification}

In a second step, we applied bootstrap aggregating (feature bagging) to identify the components that best separate between the two groups (Fig. 2A). We drew a random subset of features $\left(n_{\text {sub }}=10\right)$ from all 1380 features, classified the two groups using a support vector machine classifier with leave-one-out cross-validation, and repeated this procedure $\left(n_{\text {draw }}=2 \times 10^{7}\right)$. As control analyses, we repeated the procedure for $n_{\text {sub }}=2,3,5,20$ and for three other classification algorithms (decision trees, linear discriminatory analysis, naïve Bayes classifier). This procedure resulted in a distribution of classification accuracies across random feature selections. We defined the features that best separate between patients and controls by applying a threshold at the 75-percentile of this distribution. If no feature can classify between the two groups the probability of any feature to be in the subsets with the $25 \%$ highest accuracies will be equal. However, if features contain information to classify the two groups, this probability will increase. Thus, we defined the probability of a feature to be in the $25 \%$ subsets with the highest accuracies as its classification score. As control analyses, we repeated the analyses for different accuracy thresholds: $66 \%, 90 \%$ and $98 \%$ percentile.

\section{Statistical testing of classification scores}

We used permutation statistics to test the statistical significance of every feature's classification score, i.e., the likelihood of a given classification score under the Nullhypothesis that groups cannot be classified based on this feature. A significant feature indicates a spatio-spectral pattern of cortical coupling that separates between patient and control groups.

For each permutation ( $n=1000$ ), we randomly reassigned subjects to one of the two groups, keeping the same ratio between groups and repeated our analysis. Importantly, for each permutation, we again drew $n_{\text {draw }}=2 \times 10^{7}$ feature bags per group permutation to account for the random separability of any group constellation. We then combined all classification scores (1000 permutations of 1380 feature-specific classification scores) to define one general, component-independent Null-distribution and to assign $p$-values for each classification score. We corrected these $p$-values with false-discovery rate correction across the 1380 scores (FDR-correction) $)^{51}$.

To test if groups could be significantly classified in general, we quantified how likely it was to find the identified number of significant components under the Null-hypothesis that groups could not be classified. We applied the same procedure as for the real data to every group permutation and quantified how many significant components we could identify $(p<0.05$, 
FDR-corrected). The resulting distribution across the 1000 permutations served as the Nulldistribution of significant components. All permutations had less significant features than identified for the real data (58 features). In fact, not more than one significant feature ( $p<$ 0.05 , FDR-corrected) was identified for any of the 1000 permutations.

\section{Classification confidence}

We quantified classification confidence as a distance measure. For each subject $i$, we compared the Mahalanobis distance $D_{\text {mah }}$ of its feature vector to the distribution of feature vectors for the patient and for the control group. We computed $D_{\text {mah }}$ of subject i:

$$
D_{M a h, i, g}=\left(f_{i}-\mu_{g}\right) * C_{g}^{-1} *\left(f_{i}-\mu_{g}\right)^{\prime}
$$

Here, $f i$ describes the feature vector of each subject; $\mu$ and $C$ describe the group $g$ mean and group member covariance, respectively. We excluded the ith subject from the corresponding mean and covariance matrix calculation. The exponential -1 indicates matrix inverse and the 'operator indicates the transpose. We then defined classification confidence as the difference between $D_{\text {Mah,i,con }}-D_{\text {mah,i,rms. }}$. Thus, a positive value indicates that a subject is closer to the patient group mean than to the control group mean and vice versa.

\section{Spatial distribution of PCA components}

The component coefficients derived from the HCP-dataset contain the spatial distribution of each principal component. However, the sign of these weights is inherently ambiguous. We achieved sign consistency across components by sign flipping those components for which the mean component scores within the patient group were smaller than in the control group. Hence, a positive component coefficient indicates that the coupling is relatively increased in patients whereas a negative coefficient indicates a coupling decrease.

We visualized the spatial distribution of PCA components as the average of all normalized significant components within a frequency band and coupling measure: delta $(2.8-3.4 \mathrm{~Hz})$, theta $(4-6.7 \mathrm{~Hz})$, alpha $(8-13 \mathrm{~Hz})$, beta $(16-27 \mathrm{~Hz})$, low gamma $(32-54 \mathrm{~Hz})$ and high gamma $(64-128 \mathrm{~Hz})$. For the normalization, we divided each component by the absolute 98-percentile across all its connections.

\section{Unbiased accuracy estimation}

To estimate the unbiased accuracy with which any new subject can be classified as patient or healthy control, we employed a second-level leave-one-out cross-validation. Leaving out each subject at a time, we applied the complete analysis pipeline outlined above, defined features spaces, and classified the left out subject. 
We generated a continuous estimate of accuracy by computing the distance of the left-out subject from the decision boundary. To compare distances across different feature space realizations, i.e. left out subjects, we defined distances in units of standard deviation. We divided the distance of the left-out subject in each feature space by the standard deviation of the distances to the decision boundary of all but the left-out subjects. The sign of the distance was set positive or negative if the subject was classified as a patient or healthy control, respectively.

Finally, we separately fit a Gaussian distribution to the patient $\left(N_{M S}\right)$ and healthy control group $\left(N_{\text {con }}\right)$ distances. We derived the unbiased sensitivity, specificity and accuracy of classification from these two Gaussians:

$$
\begin{gathered}
\text { sensitivity }=\int_{0}^{+\infty} N_{\text {rrms }} / \int_{-\infty}^{+\infty} N_{r r m s} \\
\text { specificity }=\int_{-\infty}^{0} N_{\text {con }} / \int_{-\infty}^{+\infty} N_{\text {con }} \\
\text { accuracy }=\frac{1}{n_{\text {all }}}\left(n_{\text {rrms }} * \text { sensitivity }+n_{\text {con }} * \text { specificity }\right)
\end{gathered}
$$

where $\int$ indicates the integral and the two Gaussians, $N_{M S}$ and $N_{\text {con }}$, are defined by the group specific mean and standard deviation. The group sizes were defined by $n_{M S}, n_{\text {con }}$ and $n_{\text {all }}$ for the patient group, the control group and all subjects, respectively.

\section{Results}

We compared brain-wide phase- and amplitude-coupling of frequency specific neuronal activity between 17 patients diagnosed with RRMS and 17 healthy control subjects. Cortical phase- and amplitude coupling was estimated from 10 min of eyes-open resting-state MEG measurements. We reconstructed cortical activity from the MEG using beamforming ${ }^{46,47}$ and quantified phase- and amplitude-coupling using the weighted phase-lag index ${ }^{49}$ and powercorrelations of orthogonalized signals ${ }^{28}$, respectively. Both measures are insensitive to volume conduction ${ }^{27,28,49}$ and show strong intra- and inter-subject reliability ${ }^{28-30,32,53}$. 


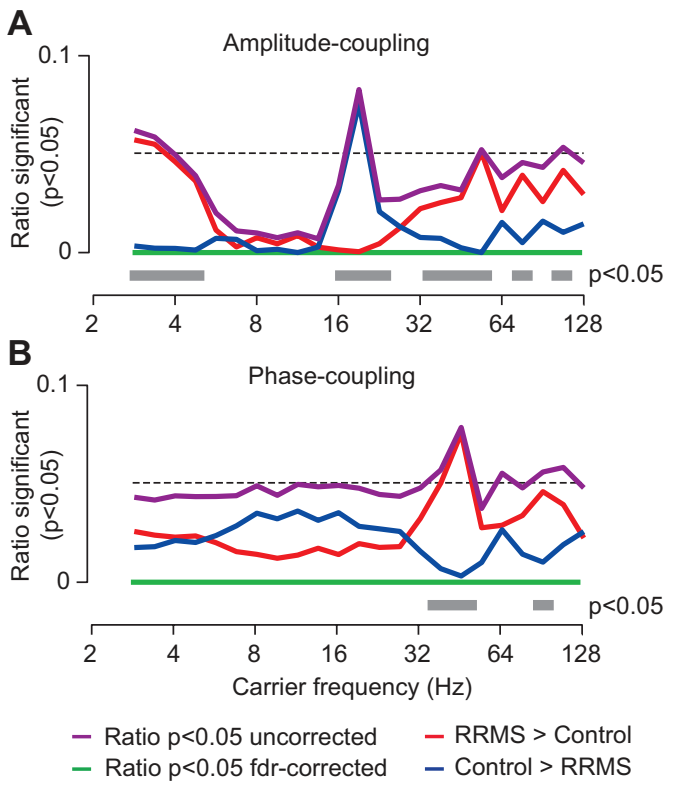

Figure 1 Connection-wise comparison of neuronal coupling between MS patients and control subjects. (A) Amplitude-coupling (orthogonalized amplitude correlations) (B) Phase-coupling (weighted phase-lag index). In both panels, the purple line indicates the ratio of significantly different connections ( $p<0.05$ uncorrected) and the green line indicates this ratio after false-discovery rate correction. The red and the blue lines show the ratio of connections with increased and decreased coupling in the patient group ( $r$ rms), respectively $(p<0.05$, uncorrected). The gray bars display carrier frequencies with a significantly directed ratio of effects $(p<0.05$, FDR-corrected), i.e., more connections with significantly increased or decreased coupling. The black dashed line indicates 0.05 .

\section{Direct comparison of neuronal coupling}

As a first approach, we directly compared the connection- and frequency-wise coupling between patients and controls (Fig. 1; $n_{c}=104,196 ; n_{f}=23$ ). While differences between groups peaked around $19 \mathrm{~Hz}$ and $45 \mathrm{~Hz}$ for amplitude- and phase-coupling, respectively (Fig. 1, purple lines), these effects were not statistically significant when applying false discovery rate-correction for the number of connections tested (Fig. 1, green lines). This highlights how the high dimensionality of brain-wide coupling impedes a direct statistical comparison.

Nevertheless, the direct comparison revealed an intriguing pattern of the sign of differences between groups (Fig. 1, red and blue lines). If there was no difference between groups, the sign of randomly significant differences in coupling (type I errors) would be equally probable in both directions. However, the observed differences deviated from this distribution for both coupling measures (Fig. 1, gray bars). For amplitude-coupling, patients showed decreased coupling in the beta frequency range $(16-22 \mathrm{~Hz})$ and increased coupling in the delta $(2-4$ $\mathrm{Hz})$ and gamma $(32-106 \mathrm{~Hz})$ frequency ranges. Phase-coupling in patients was increased in the low gamma frequency range $(38-54 \mathrm{~Hz})$. 
In summary, although the vast number of connections impaired the direct comparison of coupling on the connection-level, the asymmetry of observed differences suggested that there are systematic and frequency-specific differences of coupling between RRMS patients and healthy control subjects.
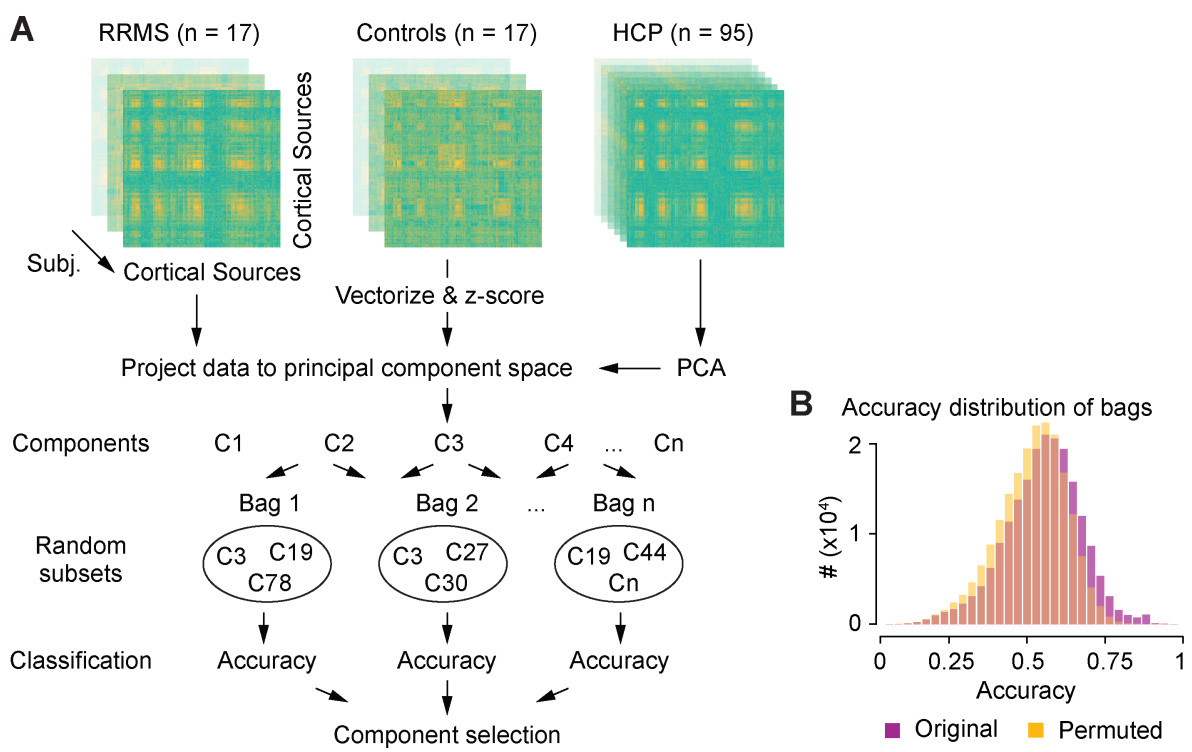

Figure 2 Analysis approach. For each subject, coupling measure and frequency we computed complete cortico-cortical correlation matrices and vectorized and z-scored the upper triangle. We conducted PCA on the coupling vectors of the HCP data and projected the coupling vectors of RRMS-patients and control subjects into the resulting space of principal coupling components. We applied bootstrap aggregating (feature bagging) to identify coupling components that best classify between RRMS-patients and control subjects. We drew random subsets of 10 components (bags) and, for each bag, classified (SVM) between groups with leave-one-out crossvalidation. We employed a permutation statistic to select components that were more often in the top quartile of best classifying bags than expected by chance (see methods) (B) Distribution of classification accuracies across all $2 \times 10^{7}$ bags for the original data (purple) and for randomly permuted group assignments of subjects (yellow).

\section{Group classification based on principal coupling components}

To overcome the limitations of the connection-wise analysis and to efficiently cope with the high dimensionality of the brain-wide coupling space, we next devised a multistage machinelearning approach (Figure 2). In brief, we first identified a subspace of principle coupling components in an independent MEG dataset, then projected the patient and control data into this coupling space, and finally employed a bootstrapped classification approach to identify significant coupling differences between groups.

To efficiently reduce the dimensionality of the coupling space, we applied principal component analysis (PCA) to the z-scored brain-wide phase- and amplitude-coupling of 95 subjects from the human connectome project S900 MEG dataset ${ }^{38}$. For each frequency and 
both coupling modes, we extracted the 30 principal coupling components that explained most variability of coupling across subjects (largest eigenvalues). Importantly, the identification of principal coupling components in an independent dataset ensured, that this identification itself was not conflated by any potential variability between patient and control groups. We next projected the coupling profiles of patients and control subjects into the principal coupling space, which resulted in a more than 3000 -fold dimensionality reduction to a total of 1380 coupling components.

A

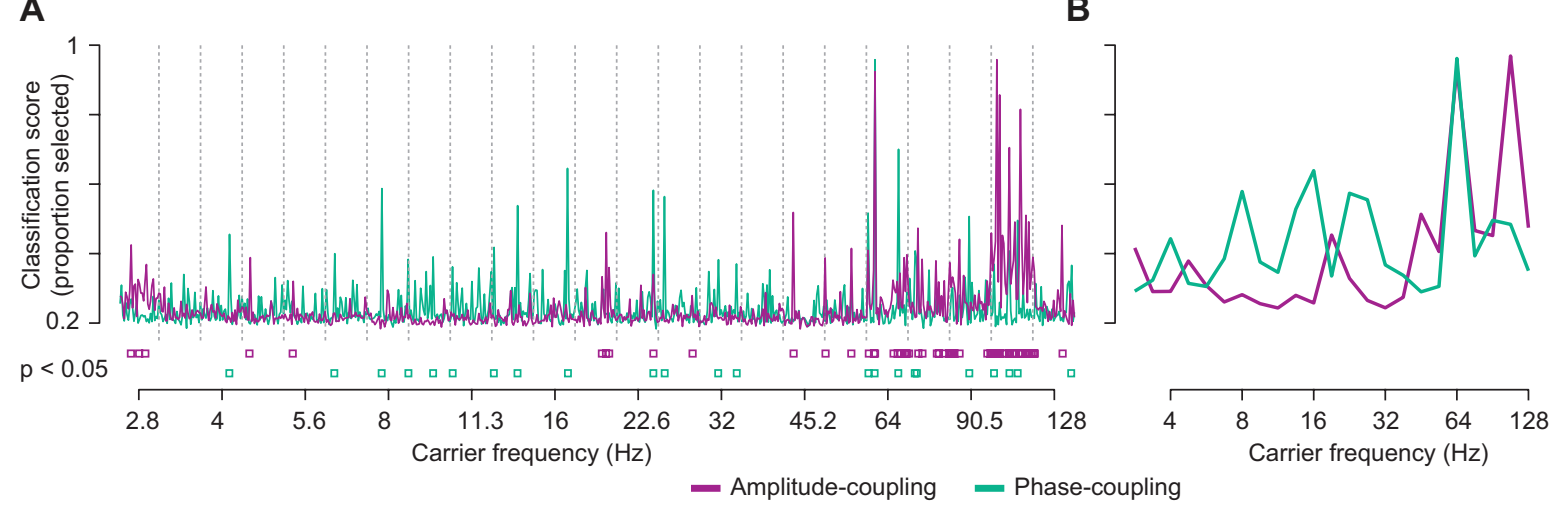

Figure 3 Selection of principal coupling components by classification scores. Classification scores were defined as the likelihood of each component to contribute to bags with classification accuracies in the top quartile of all $2 \times 10^{7}$ bags. (A) Classification scores for all 30 amplitude- (purple) and phase-coupling (green) components of each carrier frequency ordered by the variance explained within each frequency. The components and eigenvalues were derived from the HCP-dataset. Dashed lines divide the carrier frequencies. Purple and green squares indicate significant classification scores for amplitude- and phase-coupling components, respectively ( $p$ $<0.05$, FDR-corrected) (B) Maximum classification scores per carrier frequency for amplitude- (purple) and phase-coupling (green).

We next employed multivariate classification (support vector machine, SVM) to identify significant differences of coupling between groups in the reduced coupling space. Because likely not all components are informative, classification accuracy suffers when utilizing all components at once ${ }^{54}$. Similarly, by using every component for itself, generic interactions between components and frequencies might be missed. We therefore employed a bootstrap approach (feature bagging). 20-million times, we picked a random subset of 10 coupling components (without replacement) and applied SVM to classify patients and controls with leave-one-out cross-validation (Fig. 2B). For each component, we then computed a classification score as its likelihood to contribute to component-subsets with classification accuracies in the top quartile (Fig. 3A). For each component, we statistically tested its classification score using a permutation approach ( $p<0.05$, FDR-corrected). This procedure allowed us to identify 58 coupling components that significantly contributed to the classification of patient and control groups (Fig. 3A, p <0.05 FDR-corrected). 34 and 24 of these components were specific to amplitude-coupling and phase-coupling, respectively. 
The employed permutation statistic also allowed us to test whether overall we could significantly classify between the two groups. We quantified how likely it was to find the identified number of significant components under the Null-hypothesis that groups could not be classified. We found that the amount of identified features was indeed highly significant ( $p$ $<0.001$ ). Thus, RRMS patients could be significantly classified from healthy control subjects based on a specific set of principal phase- and amplitude-coupling components.

These results were robust across a broad range of parameter choices and control analyses. First, we repeated the analysis separately for amplitude- and phase-coupling components. The classification scores were similar and well correlated to those obtained when combining amplitude- and phase-coupling components (amplitude-coupling $r=0.79$; phase-coupling: $r$ $=0.65)$. We further repeated the analyses for different bag sizes $(2,3,5,20)$, accuracy thresholds $(0.66,0.9,0.98)$, and classification algorithms (naïve Bayes, decision tree and linear discriminatory analysis). The results were again very similar with high correlations of classification scores between approaches (bag size: $0.92<r<0.96$; accuracy threshold: $0.81<r<0.99$; algorithm: $0.65<r<0.91$; all $p<0.05$, FDR-corrected across all comparisons).

\section{Spectral and cortical distribution of altered coupling}

We further examined the spectral and cortical distribution of coupling components that dissociated RRMS patients from healthy controls. For amplitude-coupling, these components were spectrally specific to low frequencies $(<5 \mathrm{~Hz})$, the beta frequency range $(19 \mathrm{~Hz})$ and the gamma frequency range (> $40 \mathrm{~Hz}$; Fig. 3B). For phase-coupling, the classification score distribution showed several peaks in the theta $(4 \mathrm{~Hz})$, alpha $(8 \mathrm{~Hz})$, beta (13 - $16 \mathrm{~Hz} \& 22-26 \mathrm{~Hz}$ ) and gamma bands (> $45 \mathrm{~Hz}$; Fig. 3B).

To visualize the brain regions whose coupling dissociated patients from control, we first averaged the absolute coupling coefficients of all significant components for each brain region for frequencies below and above $35 \mathrm{~Hz}$ and both coupling measures (Fig. 4A). We split these frequency ranges because at frequencies above $35 \mathrm{~Hz}$ coupling estimated from MEG strongly resembles residual muscle activity ${ }^{30,41}$.

We found that, for frequencies below $35 \mathrm{~Hz}$, RRMS affected intra- and interhemispheric amplitude-coupling of the medial prefrontal, dorsolateral prefrontal, pericentral, lateral parietal and extrastriate visual cortex (Fig. 4A). Phase-coupling differences in this frequency range between patients and controls peaked in bilateral pericentral, inferior temporal and medial occipito-parietal areas, mainly with altered intra-hemispheric coupling. For high frequencies above $35 \mathrm{~Hz}$, for both coupling modes, we found differences between patients 
bioRxiv preprint doi: https://doi.org/10.1101/2021.02.17.431597; this version posted February 18, 2021. The copyright holder for this preprint (which was not certified by peer review) is the author/funder, who has granted bioRxiv a license to display the preprint in perpetuity. It is made available under aCC-BY-NC-ND 4.0 International license.

and controls for areas typically related to residual muscle-activity, that is bilateral anterior temporal and ventral frontal regions (Fig. 4A).

A

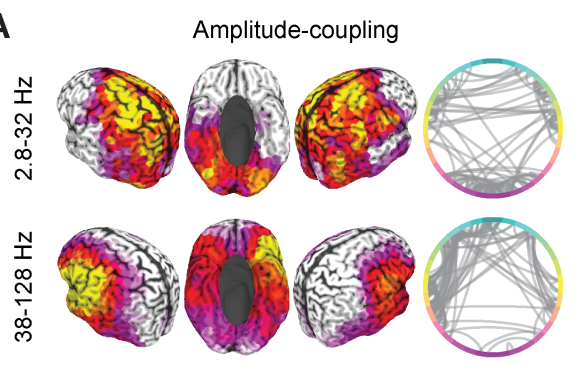

B
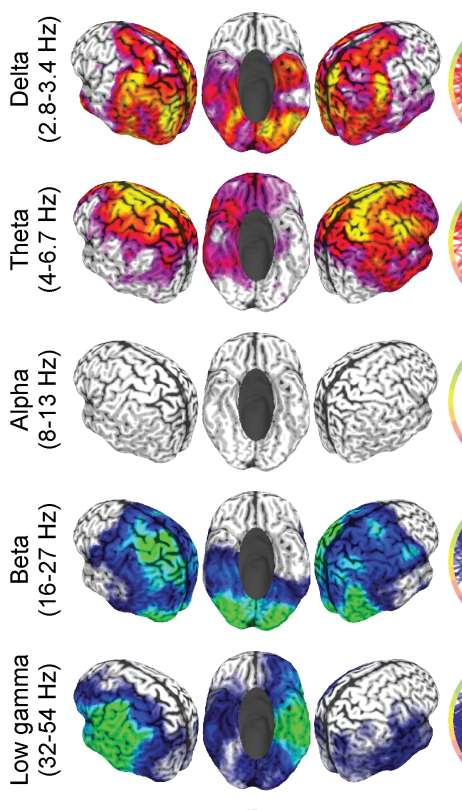

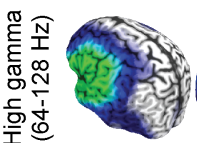
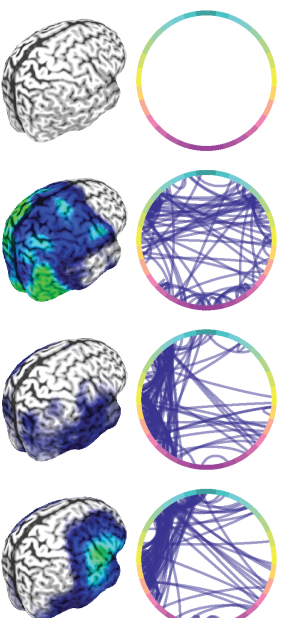
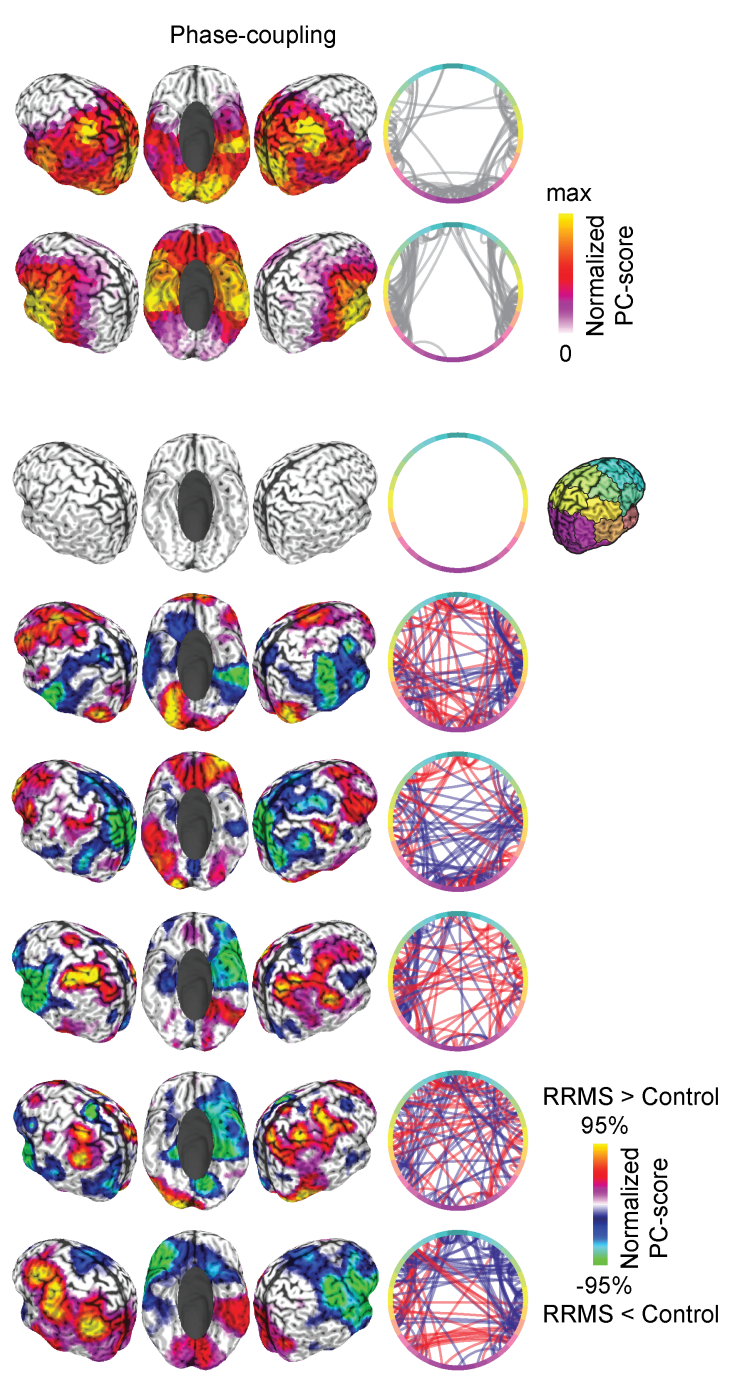

Figure 4 Cortical distribution of classifying coupling components. (A) Normalized average absolute strength of significantly classifying components for amplitude- (left) and phase-coupling (right), and for frequencies below (top) and above (bottom) $35 \mathrm{~Hz}$. Colors are scaled between 0 and maximum for each panel. Circular line plots indicate connections with best classification between patients and controls (random subset of best $10 \%$ ). Colors along the circle indicate cortical location, as shown on the right. (B) Normalized average strength of significantly classifying components for individual frequency bands and both coupling modes. Colors are scaled between the positive and negative absolute 95-percentile for each panel. Warm and cold colors indicate an increased and decreased coupling in patients, respectively. Circular line plots as in (A).

We next resolved the signed changes of coupling in patients within each frequency band (Fig. 4B). For amplitude-coupling, patients showed enhanced low-frequency coupling in lateral parietal and extrastriate visual areas in the delta band $(2.8-3.4 \mathrm{~Hz})$ and in medial and ventrolateral prefrontal cortex in the theta band $(4-6.7 \mathrm{~Hz})$. In the beta band $(16-27 \mathrm{~Hz})$, patients showed reduced amplitude-coupling in pericentral and visual areas. Frequencies above $32 \mathrm{~Hz}$ showed reduced amplitude-coupling in often muscle confounded anterior 
temporal and ventral prefrontal regions. Phase-coupling showed a rich pattern of changes in MS patients with typically bilateral increases and decreases of coupling within the same frequency range. Patients showed increased phase-coupling in medial prefrontal (theta, 4 $6.7 \mathrm{~Hz}$ ), lateral prefrontal (theta, alpha, beta \& low gamma $8-54 \mathrm{~Hz}$ ), as well as pericentral and lateral parietal areas (beta \& gamma, $16-128 \mathrm{~Hz}$ ). Phase-coupling was decreased in temporal (theta $4-6.7 \mathrm{~Hz}$ ), medial and lateral parietal (alpha $8-13 \mathrm{~Hz}$ ) as well anterior temporal areas (beta \& gamma $16-128 \mathrm{~Hz}$ ).

In summary, RRMS patients showed both, increased and decreased phase- and amplitudecoupling across a broad range of frequencies and cortical regions with highly frequencyspecific changes.

\section{Altered coupling predicts disease severity}

If the identified changes of neuronal coupling in MS patients reflected disease-specific mechanisms, they may predict disease severity. Our results supported this hypothesis. We tested if, across patients, the confidence of classification based on neuronal coupling, i.e. how similar coupling was to that of patients as compared to controls, was correlated with two clinical measures of disease strength: the Expanded Disability Status Scale ${ }^{36}$ and the Multiple Sclerosis Functional Composite ${ }^{37}$. We found that indeed both clinical scores were significantly correlated with classification confidence (Fig. 5) (EDSS: $r=0.45, p=0.04$, FDRcorrected; MSFC: $r=0.49, p=0.02$, FDR-corrected). Thus, stronger coupling changes predicted a more severe disease state.

A

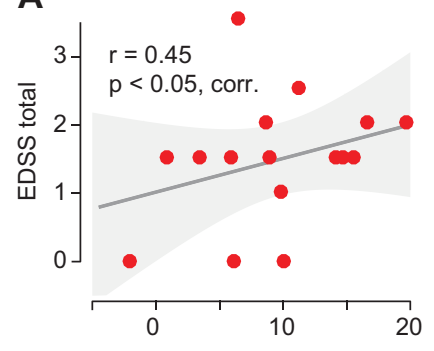

B

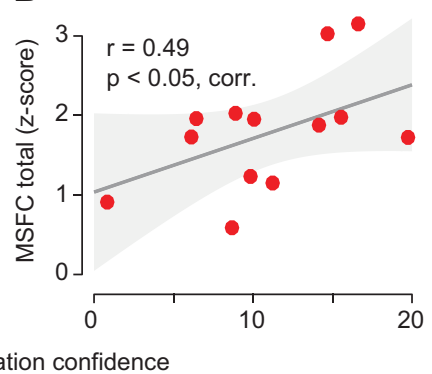

Figure 5 Correlations between classification confidence and behavioral scores of disease severity. Correlation between classification confidence and (A) the EDSS (Expanded Disability Status Scale) and (B) the MSFC-scores (Multiple Sclerosis Functional Composite). The y-axes indicate the disease severity such that an increasing value corresponds to a more severe disease state. For MSFC we mirrored the values into the positive range to fit this notation (original values $\mathrm{MSFC}_{z}=-0.3-3.3$ ). Shaded areas are $95 \%$ confidence intervals of the linear model parameters. 


\section{Classification accuracy}

In a final set of analyses, we quantitatively addressed the question how well RRMS patients could be classified based on the cortical coupling assessed with MEG. Importantly, the classification accuracy of an individual subject based on the principal coupling components identified including this subject is positively biased. Thus, to derive an unbiased estimate of classification accuracy for a novel MEG dataset, we performed a cross-validation of the entire analysis pipeline leaving out and classifying each subject at a time. We performed this analysis for all combined coupling components $\left(n_{\text {comp }}=1380\right)$ as well as separately for the two coupling modes $\left(n_{\text {comp }}=690\right)$ and frequency ranges $\left(n_{\text {comp,low }}=480 \& n_{\text {comp, high }}=210\right)$.

A

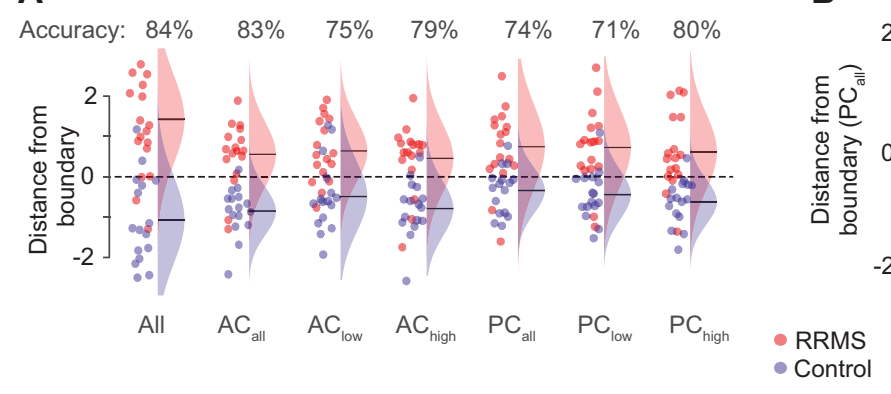

B

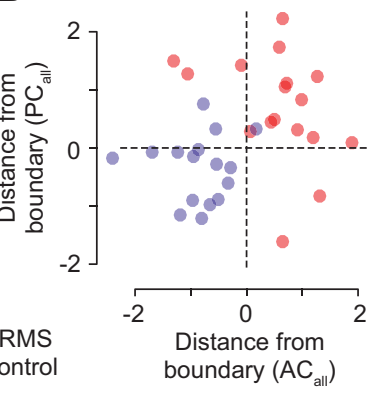

Figure 6 Classification accuracy. (A) Distance of every subject from the decision boundary (classification confidence) and Gaussian fits of the group distributions. Distances are normalized by the standard deviation across all subjects within each cross-validation fold. The circle color indicates the group; red for RRMS-patients and blue for controls. Frequencies are split at $35 \mathrm{~Hz}$ (low vs. high). (B) Each subjects' distance from the classification boundary for all amplitude- and phase-coupling components. AC (amplitude-coupling); PC (phasecoupling).

The unbiased classification accuracy using all coupling components was $84 \%$ (chance level: $50 \%$ ) and nominally higher than the accuracy obtained using any coupling mode or frequency range alone (Fig. 6A). The independent classification accuracy for amplitudecoupling (83\%) was higher than for phase-coupling (74\%). Furthermore, we found that the distance to the classification boundary was only weakly correlated between amplitude- and phase-coupling $(r=0.21)$ (Fig. $6 \mathrm{~B})$. In line with the enhanced accuracy when combining both coupling modes, this is supporting evidence for non-redundant information of phase- and amplitude-coupling. 


\section{Discussion}

This study provides, to our knowledge, the first systematic comparison of brain-wide phaseand amplitude-coupling in Multiple Sclerosis patients. We identified several principal coupling components, which could significantly classify patients from healthy controls with an overall accuracy of $84 \%$ and with classification confidence predicting disease severity. For both coupling modes, in RRMS patients we found both, increased and decreased coupling across a broad range of frequencies and cortical regions. At higher frequencies, effects likely at least partially reflected changes in muscle activity ${ }^{30,41}$. In summary, our results show systematic, wide-spread and non-redundant changes of phase- and amplitudecoupling in Multiple Sclerosis.

\section{Classification approach}

We employed a novel unsupervised multivariate classification approach that combines several key methodological advantages. First, all analyses were carried out on reconstructed cortical activity, rather than on the raw MEG-signals. This allowed to align subjects and enhanced the signal-to-noise ratio by rejecting non-neuronal activity and focusing on signals of specific neuronal origin ${ }^{41,47}$. Furthermore, we employed phase- and amplitude-coupling measures that rejected spurious coupling due to field spread.

Second, we combined dimensionality reduction and bootstrap aggregating to classify between groups in a very high-dimensional dataspace. Critically, the dimensionality reduction was based on a large and completely independent dataset. Thus, the identified principal coupling components are reflecting universal characteristics of cortical coupling across human subjects independent from the variance between the two groups at hand. In turn, the identified components can be readily applied to any new dataset and question at hand. We employed a bootstrap approach (feature bagging) ${ }^{55}$ to identify several relevant dimensions in the reduced dataspace. The results were similar across a broad range of bootstrap parameters (bag size, threshold) and classification algorithms, supporting the robustness and broad applicability of this approach.

Third, cross-validation at two critical stages ensured the generalization of results at the population level. First, we cross-validated (leave-one-out) the classification between groups for every bootstrap aggregate of coupling components. This prevented overfitting for the identification of classifying coupling components. Second, we cross-validated (leave-oneout) the classification accuracy of the entire analysis pipeline, which ensured an externally valid and unbiased estimate of the classification accuracy for new datasets. 
High dimensional feature spaces are a common problem in brain-connectivity analyses and biomedical research in general. Classification of groups of samples on the entire space is often not feasible because of uninformative dimensions and classification on individual dimensions may be computationally impractical, might miss multivariate interactions, and is difficult to control for multiple comparisons ${ }^{25,54,56}$. In the present case, this is well illustrated by the failure to detect significant coupling changes in a direct connection-level comparison. In these situations, the new analyses approach employed here provides a flexible tool that can be readily adapted to classify comparably small samples in a high-dimensional space.

\section{Wide-spread bidirectional changes of cortical coupling}

Our results provide new insights into the spatial and spectral distribution of cortical coupling changes during RRMS. We found altered coupling across the entire investigated frequency range $(2.8$ to $128 \mathrm{~Hz}$ ). This adds to a growing but heterogenous body of studies that have identified MS-related changes of rhythmic neural activity or coupling across various different frequency bands ${ }^{7,13,16-18,57-60}$. For phase-coupling, our approach revealed both increases and decreases within the same frequency range. This may have contributed to the heterogeneity of previous findings and highlights the advantage of separating cortical networks at the source-level.

Rhythmic coupling at different frequencies reflects interactions in specific neuronal microand macro-circuits ${ }^{24,61}$. The spectrally widespread nature of our findings suggests that MS leads to alterations across many different circuit interactions. This may entail not only local and large-scale cortico-cortical interactions, but also cortico-subcortical interactions. For example, the altered phase-coupling in the alpha frequency range found here may reflect altered cortico-thalamic interactions associated with MS-related thalamic atrophy ${ }^{17,59}$.

The observed broad spatial distribution of altered coupling across the cortex accords well with its broad spectral distribution. Changes involved both, local and large-scale coupling. In general, the affected cortical networks resembled the known association with specific frequency ranges, such as altered theta- or beta- coupling in midfrontal and sensorimotor regions, respectively. This suggests that RRMS alters the strength of interactions in common brain networks associated with specific frequencies. It remains to be determined, to what extent also the frequency of these interactions is affected ${ }^{59}$.

At frequencies above $35 \mathrm{~Hz}$, RRMS patients showed decreased frontotemporal coupling, which well resembled the reported distribution of residual muscle activity in EEG and $M_{E G}{ }^{30,41}$. Thus, the decreased high-frequency coupling may be due to decreased muscle activity, which may in turn result from both, the disease itself as well as from motivational differences between patients and control subjects. 
Motivational or cognitive differences may generally contribute to classification results between patient and control groups. In this context, the finding that classification confidence predicts disease severity within the patient groups is particularly important. This finding suggests that the identified changes indeed reflect disease specific effects rather than general motivational differences between patient and control groups.

Several different mechanisms may contribute to such disease specific changes. On the one hand, lesions of white-matter tracts may directly cause a reduced coupling of the connected neuronal populations. On the other hand, such reduced anatomical connectivity and functional coupling may induce a number of indirect effects. For example, the decoupled populations may be part of a larger neuronal network. Local decoupling in this network may lead to a global decrease of coupling, compensatory enhancement of coupling or a mere shift of the network dynamics. Similarly, changes within one network may again lead to both, decreases as well as increases of coupling in other brain networks $s^{6,7,11,62,63}$. Importantly, all these changes could span a broad range of temporal-scales, which may even lead to opposite immediate and long-term effects of the disease.

The interplay of all these mechanisms may explain the wide-spread, complex and often bidirectional pattern of coupling changes observed here. The present results set the stage for future studies to disentangle the different mechanisms underlying these changes. For this, longitudinal investigations will be particularly important ${ }^{7}$.

\section{Coupling mode specific changes}

Amplitude correlation of orthogonalized signals has recently been introduced as a robust and spectrally specific marker of cortical coupling ${ }^{28,30,32}$. Consistent with other recent evidence $^{21}$, our results show that cortical amplitude-coupling is systematically altered in RRMS patients already at an early disease stage. Moreover, our findings uncover markedly distinct changes for phase- and amplitude-coupling. There were more coupling components that dissociated the groups and higher classification accuracy for amplitude-coupling than for phase-coupling (amplitude/phase-coupling: 34/24 components; 83/74\% accuracy). This suggests that coupling changes are more robust for amplitude-coupling.

Furthermore, while phase-coupling showed effects in all but the delta-band, amplitudecoupling was altered in all but the alpha-band. Effects were also spatially dissociated. Below $35 \mathrm{~Hz}$, amplitude-coupling showed strongest changes in medial and lateral prefrontal cortex as well as in pericentral and medial parietal areas, while phase-coupling showed strongest effects in pericentral, medial occipitoparietal and inferior temporal cortex. Additionally, while amplitude-coupling showed either consistent increases or decreases of coupling within each band, phase-coupling showed bidirectional effects within each band. For frequencies above 
$35 \mathrm{~Hz}$, amplitude-coupling showed higher sensitivity to residual muscle activity. Finally, we found that the two coupling modes showed different sensitivities to different subgroups of subjects.

Overall, our results show that phase- and amplitude-coupling are sensitive to at least partially distinct changes of cortical coupling in MS. Accordingly, while both coupling modes could independently dissociate patients from healthy controls, the combination of coupling modes increased classification accuracy. Our findings suggest that amplitude-coupling provides a robust biomarker of changes in large-scale network dynamics during RRMS that may be synergistically combined with phase-coupling measures. Furthermore, the observed differences between coupling modes adds to converging evidence that at least partially distinct neuronal mechanisms underlie both coupling modes ${ }^{24,31,32,50}$.

\section{Summary and conclusions}

In summary, we devised a new analysis approach that combines dimensionality reduction and bootstrapped multivariate classification to identify disease-related neuronal coupling changes. Our approach can be readily adapted to other scientific questions, and thus, holds great potential for the comparison of groups in high-dimensional search spaces.

Our results uncover systematic changes of large-scale cortical phase- and amplitudecoupling at an early disease stage of Multiple Sclerosis. Changes were coupling-mode specific and included decreases as well as increases across wide-spread frequency ranges and cortical networks. Our results highlight non-invasive electrophysiological measures of neuronal coupling as powerful new biomarkers of relapsing-remitting Multiple Sclerosis. 


\section{Acknowledgements}

We thank Hannah Krämer, Yeo Kim and Gabi Walker-Dietrich for help with data acquisition.

\section{Data availability statement}

The data is available from the authors upon reasonable request.

\section{Funding}

This work was supported by the European Research Council (ERC) StG335880 (M.S) and the Centre for Integrative Neuroscience (DFG, EXC 307) (M.S.). The authors acknowledge support by the state of Baden-Württemberg through bwHPC, the German Research Foundation (DFG) through grant no INST 39/963-1 FUGG (bwForCluster NEMO), and a grant from Biogen Idec GmbH (U.Z.).

\section{Competing interests}

The authors declare no competing financial interests. 


\section{References}

1. Dendrou CA, Fugger L, Friese MA. Immunopathology of multiple sclerosis. Nat Rev Immunol. 2015;15(9):545-558. doi:10.1038/nri3871

2. Amato MP, Portaccio $E$, Goretti $B$, et al. Cognitive impairment in early stages of multiple sclerosis. Neurol Sci. 2010;31(2):211-214. doi:10.1007/s10072-010-0376-4

3. Chiaravalloti ND, DeLuca J. Cognitive impairment in multiple sclerosis. Lancet Neurol. 2008;7(12):1139-1151. doi:10.1016/S1474-4422(08)70259-X

4. Benedict RHB, Weinstock-Guttman B, Fishman I, Sharma J, Tjoa CW, Bakshi R. Prediction of Neuropsychological Impairment in Multiple Sclerosis: Comparison of Conventional Magnetic Resonance Imaging Measures of Atrophy and Lesion Burden. Arch Neurol. 2004;61(2):226. doi:10.1001/archneur.61.2.226

5. Benedict RHB, Zivadinov R. Risk factors for and management of cognitive dysfunction in multiple sclerosis. Nat Rev Neurol. 2011;7(6):332-342. doi:10.1038/nrneurol.2011.61

6. Stam CJ. Modern network science of neurological disorders. Nat Rev Neurosci. 2014;15(10):683-695. doi:10.1038/nrn3801

7. Schoonheim MM, Meijer KA, Geurts JJG. Network Collapse and Cognitive Impairment in Multiple Sclerosis. Front Neurol. 2015;6. doi:10.3389/fneur.2015.00082

8. Roosendaal SD, Geurts JJG, Vrenken H, et al. Regional DTI differences in multiple sclerosis patients. Neurolmage. 2009;44(4):1397-1403. doi:10.1016/j.neuroimage.2008.10.026

9. Shu N, Liu Y, Li K, et al. Diffusion Tensor Tractography Reveals Disrupted Topological Efficiency in White Matter Structural Networks in Multiple Sclerosis. Cereb Cortex. 2011;21(11):2565-2577. doi:10.1093/cercor/bhr039

10. Tewarie P, Steenwijk MD, Tijms BM, et al. Disruption of structural and functional networks in long-standing multiple sclerosis. Hum Brain Mapp. 2014;35(12):59465961. doi:10.1002/hbm.22596

11. Helekar SA, Shin J, Mattson BJ, et al. Functional Brain Network Changes Associated with Maintenance of Cognitive Function in Multiple Sclerosis. Front Hum Neurosci. 2010;4. doi:10.3389/fnhum.2010.00219

12. Hawellek DJ, Hipp JF, Lewis CM, Corbetta M, Engel AK. Increased functional connectivity indicates the severity of cognitive impairment in multiple sclerosis. Proc Natl Acad Sci. 2011;108(47):19066-19071. doi:10.1073/pnas.1110024108 
13. Hardmeier M, Schoonheim MM, Geurts JJG, et al. Cognitive dysfunction in early multiple sclerosis: altered centrality derived from resting-state functional connectivity using magneto-encephalography. PloS One. 2012;7(7):e42087.

doi:10.1371/journal.pone.0042087

14. Rocca MA, Valsasina $P$, Martinelli $V$, et al. Large-scale neuronal network dysfunction in relapsing-remitting multiple sclerosis. Neurology. 2012;79(14):14491457. doi:10.1212/WNL.0b013e31826d5f10

15. Rocca MA, Valsasina P, Hulst HE, et al. Functional correlates of cognitive dysfunction in multiple sclerosis: A multicenter fMRI Study. Hum Brain Mapp. 2014;35(12):5799-5814. doi:10.1002/hbm.22586

16. Schoonheim MM, Geurts JJG, Landi D, et al. Functional connectivity changes in multiple sclerosis patients: A graph analytical study of MEG resting state data. Hum Brain Mapp. 2013;34(1):52-61. doi:10.1002/hbm.21424

17. Tewarie P, Schoonheim MM, Stam CJ, et al. Cognitive and Clinical Dysfunction, Altered MEG Resting-State Networks and Thalamic Atrophy in Multiple Sclerosis. PLoS ONE. 2013;8(7):e69318. doi:10.1371/journal.pone.0069318

18. Tewarie $P$, Hillebrand $A$, Schoonheim MM, et al. Functional brain network analysis using minimum spanning trees in Multiple Sclerosis: An MEG source-space study. Neurolmage. 2014;88:308-318. doi:10.1016/j.neuroimage.2013.10.022

19. Cogliati Dezza I, Zito G, Tomasevic L, et al. Functional and structural balances of homologous sensorimotor regions in multiple sclerosis fatigue. J Neurol. 2015;262(3):614-622. doi:10.1007/s00415-014-7590-6

20. Gschwind M, Hardmeier M, Van De Ville D, et al. Fluctuations of spontaneous EEG topographies predict disease state in relapsing-remitting multiple sclerosis. Neurolmage Clin. 2016;12:466-477. doi:10.1016/j.nicl.2016.08.008

21. Sjøgård $M$, Wens $\mathrm{V}$, Schependom JV, et al. Brain dysconnectivity relates to disability and cognitive impairment in multiple sclerosis. Hum Brain Mapp. 2021;42(3):626-643. doi:https://doi.org/10.1002/hbm.25247

22. Kocevar G, Stamile C, Hannoun S, et al. Graph Theory-Based Brain Connectivity for Automatic Classification of Multiple Sclerosis Clinical Courses. Front Neurosci. 2016;10. doi:10.3389/fnins.2016.00478

23. Liu $\mathrm{Y}$, Wang $\mathrm{H}$, Duan $\mathrm{Y}$, et al. Functional Brain Network Alterations in Clinically Isolated Syndrome and Multiple Sclerosis: A Graph-based Connectome Study. Radiology. 2016;282(2):534-541. doi:10.1148/radiol.2016152843 
24. Siegel M, Donner TH, Engel AK. Spectral fingerprints of large-scale neuronal interactions. Nat Rev Neurosci. 2012;13(2):121-134. doi:10.1038/nrn3137

25. Hipp JF, Siegel M. BOLD fMRI Correlation Reflects Frequency-Specific Neuronal Correlation. Curr Biol CB. 2015;25(10):1368-1374. doi:10.1016/j.cub.2015.03.049

26. Fries $P$. A mechanism for cognitive dynamics: neuronal communication through neuronal coherence. Trends Cogn Sci. 2005;9(10):474-480.

27. Brookes MJ, Woolrich MW, Barnes GR. Measuring functional connectivity in MEG: a multivariate approach insensitive to linear source leakage. Neurolmage. 2012;63(2):910-920. doi:10.1016/j.neuroimage.2012.03.048

28. Hipp JF, Hawellek DJ, Corbetta M, Siegel M, Engel AK. Large-scale cortical correlation structure of spontaneous oscillatory activity. Nat Neurosci. 2012;15(6):884-890. doi:10.1038/nn.3101

29. Wens V, Bourguignon M, Goldman S, et al. Inter- and intra-subject variability of neuromagnetic resting state networks. Brain Topogr. 2014;27(5):620-634. doi:10.1007/s10548-014-0364-8

30. Siems M, Pape A-A, Hipp JF, Siegel M. Measuring the cortical correlation structure of spontaneous oscillatory activity with EEG and MEG. Neurolmage. 2016;129:345-355. doi:10.1016/j.neuroimage.2016.01.055

31. Daffertshofer A, Ton R, Kringelbach ML, Woolrich M, Deco G. Distinct criticality of phase and amplitude dynamics in the resting brain. Neurolmage. 2018;180:442447. doi:10.1016/j.neuroimage.2018.03.002

32. Siems M, Siegel M. Dissociated neuronal phase- and amplitude-coupling patterns in the human brain. Neurolmage. Published online January 11, 2020:116538. doi:10.1016/j.neuroimage.2020.116538

33. Hipp JF, Engel AK, Siegel M. Oscillatory synchronization in large-scale cortical networks predicts perception. Neuron. 2011;69(2):387-396.

doi:10.1016/j.neuron.2010.12.027

34. Lublin FD, Reingold SC. Defining the clinical course of multiple sclerosis. Neurology. 1996;46(4):907. doi:10.1212/WNL.46.4.907

35. Polman $\mathrm{CH}$, Reingold SC, Banwell $\mathrm{B}$, et al. Diagnostic criteria for multiple sclerosis: 2010 Revisions to the McDonald criteria. Ann Neurol. 2011;69(2):292-302. doi:https://doi.org/10.1002/ana.22366

36. Kurtzke JF. Rating neurologic impairment in multiple sclerosis. Neurology. 1983;33(11):1444. doi:10.1212/WNL.33.11.1444 
37. Fischer JS, Rudick RA, Cutter GR, Reingold SC. The Multiple Sclerosis Functional Composite measure (MSFC): an integrated approach to MS clinical outcome assessment. Mult Scler J. 1999;5(4):244-250.

doi:10.1177/135245859900500409

38. Larson-Prior LJ, Oostenveld R, Della Penna S, et al. Adding dynamics to the Human Connectome Project with MEG. Neurolmage. 2013;80:190-201. doi:10.1016/j.neuroimage.2013.05.056

39. Van Essen DC, Smith SM, Barch DM, Behrens TEJ, Yacoub E, Ugurbil K. The WU-Minn Human Connectome Project: An overview. Neurolmage. 2013;80:62-79. doi:10.1016/j.neuroimage.2013.05.041

40. Hyvärinen A, Oja E. Independent component analysis: algorithms and applications. Neural Netw Off J Int Neural Netw Soc. 2000;13(4-5):411-430.

41. Hipp JF, Siegel M. Dissociating neuronal gamma-band activity from cranial and ocular muscle activity in EEG. Front Hum Neurosci. 2013;7:338.

doi:10.3389/fnhum.2013.00338

42. Chaumon M, Bishop DVM, Busch NA. A practical guide to the selection of independent components of the electroencephalogram for artifact correction. $J$ Neurosci Methods. 2015;250:47-63. doi:10.1016/j.jneumeth.2015.02.025

43. Mantini D, Penna SD, Marzetti L, et al. A Signal-Processing Pipeline for Magnetoencephalography Resting-State Networks. Brain Connect. 2011;1(1):49-59. doi:10.1089/brain.2011.0001

44. Oostenveld R, Fries P, Maris E, Schoffelen J-M. FieldTrip: Open Source Software for Advanced Analysis of MEG, EEG, and Invasive Electrophysiological Data. Comput Intell Neurosci. 2010;2011:e156869. doi:10.1155/2011/156869

45. Nolte $\mathrm{G}$. The magnetic lead field theorem in the quasi-static approximation and its use for magnetoencephalography forward calculation in realistic volume conductors. Phys Med Biol. 2003;48(22):3637. doi:10.1088/0031-9155/48/22/002

46. Gross J, Kujala J, Hamalainen M, Timmermann L, Schnitzler A, Salmelin R. Dynamic imaging of coherent sources: Studying neural interactions in the human brain. Proc Natl Acad Sci U S A. 2001;98(2):694-699. doi:10.1073/pnas.98.2.694

47. Van Veen BD, van Drongelen W, Yuchtman M, Suzuki A. Localization of brain electrical activity via linearly constrained minimum variance spatial filtering. IEEE Trans Biomed Eng. 1997;44(9):867-880. doi:10.1109/10.623056 
48. Goupillaud P, Grossmann A, Morlet J. Cycle-octave and related transforms in seismic signal analysis. Geoexploration. 1984;23(1):85-102. doi:10.1016/00167142(84)90025-5

49. Vinck M, Oostenveld R, van Wingerden M, Battaglia F, Pennartz CMA. An improved index of phase-synchronization for electrophysiological data in the presence of volume-conduction, noise and sample-size bias. Neurolmage. 2011;55(4):1548-1565. doi:10.1016/j.neuroimage.2011.01.055

50. Engel AK, Gerloff C, Hilgetag CC, Nolte G. Intrinsic coupling modes: multiscale interactions in ongoing brain activity. Neuron. 2013;80(4):867-886.

doi:10.1016/j.neuron.2013.09.038

51. Benjamini $Y$, Hochberg Y. Controlling the False Discovery Rate: A Practical and Powerful Approach to Multiple Testing. J R Stat Soc Ser B Methodol. 1995;57(1):289-300.

52. Wens V, Marty B, Mary A, et al. A geometric correction scheme for spatial leakage effects in MEG/EEG seed-based functional connectivity mapping. Hum Brain Mapp. 2015;36(11):4604-4621. doi:10.1002/hbm.22943

53. Colclough GL, Woolrich MW, Tewarie PK, Brookes MJ, Quinn AJ, Smith SM. How reliable are MEG resting-state connectivity metrics? Neurolmage. 2016;138:284-293. doi:10.1016/j.neuroimage.2016.05.070

54. Pappu V, Pardalos PM. High-Dimensional Data Classification. In: Aleskerov F, Goldengorin B, Pardalos PM, eds. Clusters, Orders, and Trees: Methods and Applications: In Honor of Boris Mirkin's 70th Birthday. Springer Optimization and Its Applications. Springer; 2014:119-150. doi:10.1007/978-1-4939-0742-7_8

55. Tin Kam Ho. The random subspace method for constructing decision forests. IEEE Trans Pattern Anal Mach Intell. 1998;20(8):832-844. doi:10.1109/34.709601

56. Wang SH, Lobier M, Siebenhühner F, Puoliväli T, Palva S, Palva JM. Hyperedge bundling: A practical solution to spurious interactions in MEG/EEG source connectivity analyses. Neurolmage. 2018;173:610-622.

doi:10.1016/j.neuroimage.2018.01.056

57. Cover KS, Vrenken H, Geurts JJG, et al. Multiple sclerosis patients show a highly significant decrease in alpha band interhemispheric synchronization measured using MEG. Neurolmage. 2006;29(3):783-788.

doi:10.1016/j.neuroimage.2005.08.048

58. Van Schependom J, Gielen J, Laton J, D'hooghe MB, De Keyser J, Nagels G. Graph theoretical analysis indicates cognitive impairment in MS stems from neural disconnection. Neurolmage Clin. 2014;4:403-410. doi:10.1016/j.nicl.2014.01.012 
59. Schoonhoven DN, Fraschini M, Tewarie $P$, et al. Resting-state MEG measurement of functional activation as a biomarker for cognitive decline in MS. Mult Scler Houndmills Basingstoke Engl. 2019;25(14):1896-1906.

doi:10.1177/1352458518810260

60. Figueroa-Vargas $\mathrm{A}$, Cárcamo $\mathrm{C}$, Henríquez-Ch $\mathrm{R}$, et al. Frontoparietal connectivity correlates with working memory performance in multiple sclerosis. Sci Rep. 2020;10(1):9310. doi:10.1038/s41598-020-66279-0

61. Donner TH, Siegel M. A framework for local cortical oscillation patterns. Trends Cogn Sci. 2011;15(5):191-199. doi:10.1016/j.tics.2011.03.007

62. Fornito A, Zalesky A, Breakspear M. The connectomics of brain disorders. Nat Rev Neurosci. 2015;16(3):159-172. doi:10.1038/nrn3901

63. Heuvel MP van den, Sporns O. A cross-disorder connectome landscape of brain dysconnectivity. Nat Rev Neurosci. 2019;20(7):435-446. doi:10.1038/s41583019-0177-6 